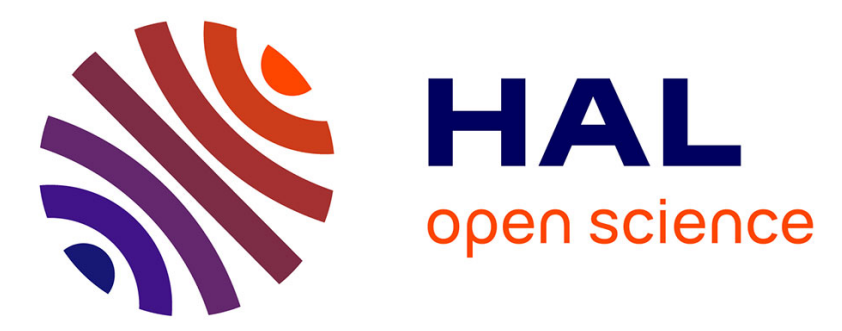

\title{
Towards Transfers in Paraplegia Assisted by Electrical Stimulation and Inertial System
}

Lucas Fonseca, Ana Claudia Lopes, Claudia Ochoa-Diaz, Christine Azevedo Coste, Emerson Fachin-Martins, Antonio Padilha Lanari Bo

\section{- To cite this version:}

Lucas Fonseca, Ana Claudia Lopes, Claudia Ochoa-Diaz, Christine Azevedo Coste, Emerson FachinMartins, et al.. Towards Transfers in Paraplegia Assisted by Electrical Stimulation and Inertial System. LSC: Life Sciences Conference, Dec 2017, Sydney, Australia. pp.292-295, 10.1109/LSC.2017.8268200 . lirmm-01630001

\section{HAL Id: lirmm-01630001 https://hal-lirmm.ccsd.cnrs.fr/lirmm-01630001}

Submitted on 5 Dec 2017

HAL is a multi-disciplinary open access archive for the deposit and dissemination of scientific research documents, whether they are published or not. The documents may come from teaching and research institutions in France or abroad, or from public or private research centers.
L'archive ouverte pluridisciplinaire HAL, est destinée au dépôt et à la diffusion de documents scientifiques de niveau recherche, publiés ou non, émanant des établissements d'enseignement et de recherche français ou étrangers, des laboratoires publics ou privés. 


\title{
Towards Transfers in Paraplegia Assisted by Electrical Stimulation and Inertial System
}

\author{
Lucas O. da Fonseca ${ }^{1}$, Ana Claudia Garcia Lopes ${ }^{2}$, Claudia Ochoa-Diaz ${ }^{1}$, \\ Christine Azevedo-Coste ${ }^{3}$, Emerson Fachin-Martins ${ }^{2}$ and Antônio P. L. Bó ${ }^{1}$
}

\begin{abstract}
Spinal cord injured (SCI) patients that have no lower limb motor function perform several transfers during a day. Those transfers are from and to a wheelchair, a car, a hygienic chair, among other situations. These repetitive motions can cause overload on their upper limbs over time. Functional Electrical Stimulation may be used to induce contraction on knee extensors, providing additional support at the joint level during transfer. However, the design of the interface with which to control the onset of stimulation is challenging. The use of some automated system is beneficial, particularly since the user is using both hands to perform the transfer. Therefore, the precise moment of activation is important because, if erroneous, it can cause the user's loss of balance. A system with which the users themselves can activate the stimulation with triggers in gloves is used to collect kinematic data from SCI patients during Sitting Pivot Transfers. The results show that the trunk angle can be used along a threshold for a reliable assistance device. Strategies for portability using a wireless inertial measurement unit are also discussed.
\end{abstract}

\section{INTRODUCTION}

A spinal cord injury (SCI) can deeply impact the individual's life and there are numerous challenges they must face everyday. Transfer from/to wheelchair is a key ability to gain mobility and independence, allowing greater interaction with the environment, besides social participation and improvements of the quality of life for individuals with spinal cord injury [9], [12], [4], [7]. The most commonly performed type of transfer by paraplegic subjects is sitting pivot transfer (SPT) [11], [3]. In most of the cases, they have potential to perform this task independently. The SPT is executed on average 15 to 20 times a day [11]. This large number of transfers also contributes to the development or perpetuation of secondary upper limbs musculoskeletal impairments over time and it is known that after SCI, individuals have great risks of pain and injury in the upper limb due to joint overloads during activities of daily living [11], [1], [2]. Functional electrical stimulation (FES) can be used as a potential technological resource to assist these people during transfers [8], [10], [6], [5]. However, how the user activates the stimulation poses a problem. Since he uses both upper limbs during the transfer, any strategy that requires manual activating at the time of stimulation is

This work was funded by Coordenação de Aperfeiçoamento de Pessoal de Nível Superior - CAPES, and Fundação de Apoio à Pesquisa - FAP/DF

${ }^{1}$ Robotics and Automation Laboratory - LARA, Electrical Engineering Department, University of Brasília - UnB

${ }^{2}$ Physiotherapy Department, Faculdade de Ceilândia, University of Brasília - UnB

${ }^{3}$ Institut de Recherche en Informatique et Automatique - INRIA, LIRMM, Montpellier, France not practical. The moment of the stimulation onset must be automated in order to free the user's upper limbs.

We hypothesized that kinematic information could be used to trigger the stimulation with minimal user training, as proposed by [5]. A possible system for FES-assisted SPT could work as follows: the user has some kind of interface, like a switch, that he uses to enter a "SPT mode" when he is already in the starting position. Then he places his hand wherever he wishes and perform the transfer. The IMU, placed over his $\mathrm{C} 7$ vertebra, captures the trunk angle and triggers the lower limbs stimulation according to the relative angle threshold previously set to that user.

We have previously performed similar tests with able-bodied subjects [8] with manual stimulation triggering. This work was motivated by the following questions: 1) can an inertial measurement unit (IMU) collect kinematic information capable of reliably trigger FES for SPT assist on paraplegic subjects? And 2) can this IMU be placed on the user such as a wearable device? In order to do that we captured kinematic data from SCI subjects performing numerous SPTs with two different systems simultaneously; an IMU and a motion capture system. In addition, we developed a glove that allowed the subject to activate the stimulation as intended during the transfer.

\section{Methods}

\section{A. Subjects and setup}

Three patients underwent the experiment: 2 men and 1 woman aging between 34 and 49 years old, all with AIS A (American Spinal Injury Association Impairment Scale) lesions between T2 and T11. They signed the Consent Form, which, along with this work, was approved by the Research and Ethics Committee (CAAE: 54748116.9.0000.0022). This work was approved by the Ethical Committee from the SARAH Network Rehabilitation Hospitals.

The patients were involved in two sessions. The first one took place at the hospital, where a physiotherapist applied a physical exam to make sure he/she was able to perform the task of SPT. The second session was the experiment in the laboratory.

Two benches were placed next to each other, with a $10^{\circ}$ angle in between. The patient's feet were positioned in front of the benches, and equidistantly to both of them. There were specific places for positioning the hands, which were kept unchanged during the whole work. The experimental set-up can be seen on Fig. 1.

In order to collect accurate kinematic data, a golden standard motion capture system was used. Also, an IMU collected 


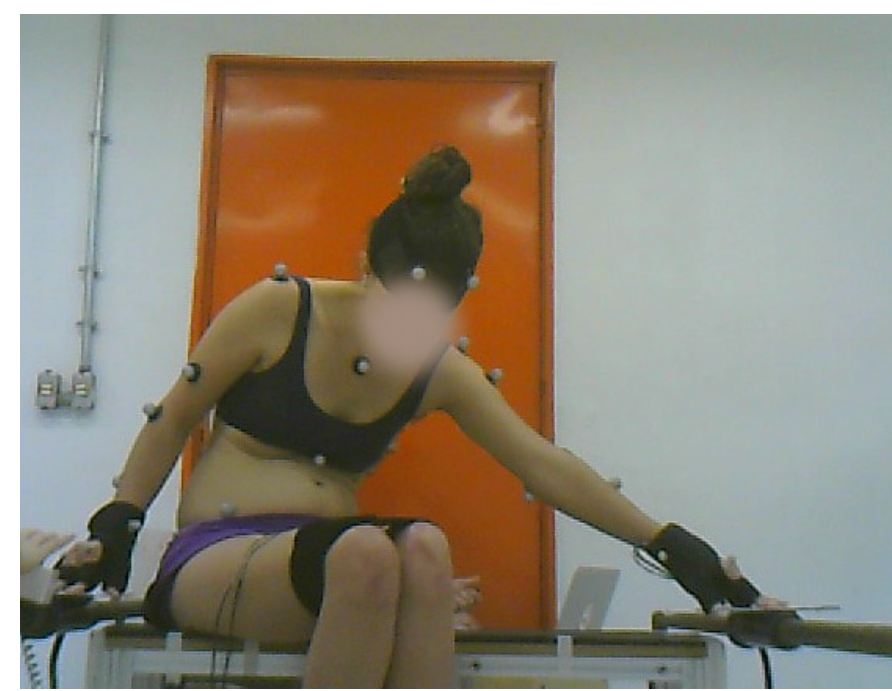

Figure 1: Experimental set-up. The gloves embed pressure sensors. It is possible to see the markers over the subject body, which are captured by the motion capture system. The benches are next to each other, with a $10^{\circ}$ angle between them. The legs are constrained to keep them for falling to the sides.

data for the purpose of this work. Finally, pressure sensors on the hands provided readings related to the pressure applied by the hand on the support.

\section{B. Stimulator}

The stimulator was a Rehastim (Hasomed, Germany). Two channels were used, one for each leg on the quadriceps muscle group. An emergency stop button was positioned on the metal structure, easily accessible by both patient and supervising physiotherapist. Stimulation was applied at $50 \mathrm{~Hz}$ and $450 \mu \mathrm{s}$. The current was determined on the day of the experiment, based on each subject's individual response to achieve leg extension for $10 \mathrm{~s}$.

\section{Hands pressure sensors}

A couple of hand pressure sensing gloves were custommade. Each glove embeds three FlexiForce sensors (Tekscan, EUA), which were connected to a microcontroller. The resistive sensors are placed on specific palm areas to maximize the force application. A microcontroller sampled data at $1 \mathrm{kHz}$ and forwarded it to a computer at $20 \mathrm{~Hz}$.

\section{Inertial measurement unit}

One inertial measurement unit (IMU, Yost Labs, EUA) was positioned over the patient's C7 vertebrae. It communicated wirelessly with the computer with a proprietary protocol and was able to sample orientation data at about $170 \mathrm{~Hz}$. In order to simulate real applications, IMUs readings were performed with respect to the initial position, i.e. angle data was always set to 0 at the start of each trial. Indeed, for everyday use, a helpful feature is possibility of using the device without any initialization, such as positioning in vertical position for providing an absolute reference. In our case, relative reference position could be obtained by high-pass filtering the measured angle.

\section{E. Motion capture system}

Qualisys QTM (Qualisys, Sweden) was used to capture motion data, as well as data from the force plates. It was all sampled at $200 \mathrm{~Hz}$ and recorded on a local computer. The upper body marker protocol ${ }^{1}$ was used with 31 markers. The C7 marker, which is usually positioned over the C7 vertebrae, was instead positioned over the IMU.

\section{F. Experimental protocol}

At the beginning of each trial, the subject was either on one bench or the other, already prepared to execute the transfer. Their hand would be placed on the predefined places, but it was asked them not to apply weight on it to prevent the stimulation activation ahead of time. The system would start collecting data, and a researcher would tell the subject they could do the transfer whenever they wanted. The subject would do it, and when they supported their weight on their upper limbs, the stimulation would be activated and cause the lower limb muscles to contract, assisting with the transfer. After the stimulation was over, and the transfer was finished, the trial was concluded.

Each subject underwent 12 transfers, 6 in each direction (left and right). Out of the total 12, 6 were performed with stimulation and 6 without it. Before the 12 transfers in which data was collected for this work, there were 6 transfers with the purpose of familiarizing the user with the system, particularly the stimulation activation method.

\section{G. Control strategy}

The hand pressure sensors gave the subjects the control over when to activate the stimulation. They could prepare themselves for the transfer and activate the stimulation when they supported their weight on their upper limbs, in a intuitive way. The stimulation then remains activated for 1 second during the lift pivot phase. The lift pivot phase is defined between the moment the subject supports his weight on the upper limbs and the moment he reaches the target seat, and it lasts on average 1 second, according to [4].

Knowing when the subject intended to activate the stimulation, we could evaluate if the wireless IMU provided kinematic data that is consistent intra-subject with the user intended stimulation activation timing. If this is indeed the case, a simpler measurement system (i.e. IMUs) could be used instead of sensorized gloves for controlling stimulation onset in FESaided SPT. This is particularly relevant for the users, who will have their upper limbs free to guide the transfer.

\section{H. Data analysis}

After the trunk angles were recorded for both the motion capture system and the IMU, intra-subject trials correlation of

\footnotetext{
${ }^{1}$ Plug-in Gait Full-Body (C-Motion, available in: https://cmotion.com/v3dwiki/index.php?title=Tutorial:_Plug-In_Gait_Full-Body)
} 
Table I: Mean and standard deviation of relative trunk angle position from the IMU data.

\begin{tabular}{|c|c|c|}
\hline Subject & Mean $\left[^{\circ}\right]$ & Standard deviation $\left[^{\circ}\right]$ \\
\hline \hline $\mathrm{A}$ & 7,74 & 2,06 \\
\hline $\mathrm{B}$ & 4,11 & 3,78 \\
\hline $\mathrm{C}$ & 5,28 & 3,31 \\
\hline
\end{tabular}

trunk angle on the sagittal plane was calculated. Then the trunk angle was analyzed along the moment in which the stimulation was activated by the subject. The same was done with the trunk angle first derivative, second derivative, relative angle and standard deviation (along a moving window of $0.1 \mathrm{~s}$ ). Since this work aims at developing wearable technologies which must work in real time, no offline non causal filters were used.

\section{RESULTS}

The trunk angle correlation between all trials in each subject presented correlation greater than 0.75 , where 1 indicates complete similarity, both with the motion capture system and the IMU data. We may infer that, even though all subjects had just been presented to a new technology and, thus, a new way of performing transfers, there was reasonable degree of similarity between the trials, concerning trunk angle on the sagittal plane. Fig. 2 shows the trunk angle on the sagittal plane on all 12 trials for one subject with the motion capture system (Fig. 2a) and the IMU (Fig. 2b). From the moment the subject was told he could do the transfer, there was no instructions for specific timing on when he should start it. Therefore, for visualization purposes, all trials were centralized by the peak trunk angle and plotted on top of each other. Also, the data was trimmed to show only the 5 seconds around the peak trunk angle. This is why not all trials start at exactly $0^{\circ}$ on Fig. $2 \mathrm{~b}$, even though the IMU angles are set to $0^{\circ}$ at the beginning.

Fig. 3 show the angles on which the stimulation was activated by one subject related to the initial angle position. Since the angles are relative to the initial position, it was expected that the activating angles would be low. Table I summarizes these relative angles means and standard deviations for the IMU data.

The first and second derivatives were calculated, but it seemed there was no simple threshold that could reliably be set to trigger the stimulation because the signal oscillated too much. The same happened with the windowed standard deviation.

\section{DISCUSSION}

The differences between the angles captured by the motion capture system, Fig. 2a, and the IMU, Fig. 2b, are due to two reasons. First, the procedure on which the IMU angles are set to $0^{\circ}$ on the beginning of each trial. Also, the two systems do not measure the exact same body segment. The trunk angle from the motion capture system is based on the trunk body segment, which was built using the shoulders and hips markers. Therefore, it does not capture the curvature along the trunk. The IMU, however, was placed over the $\mathrm{C} 7$ vertebra. As a result, the IMU captures the upper trunk orientation,

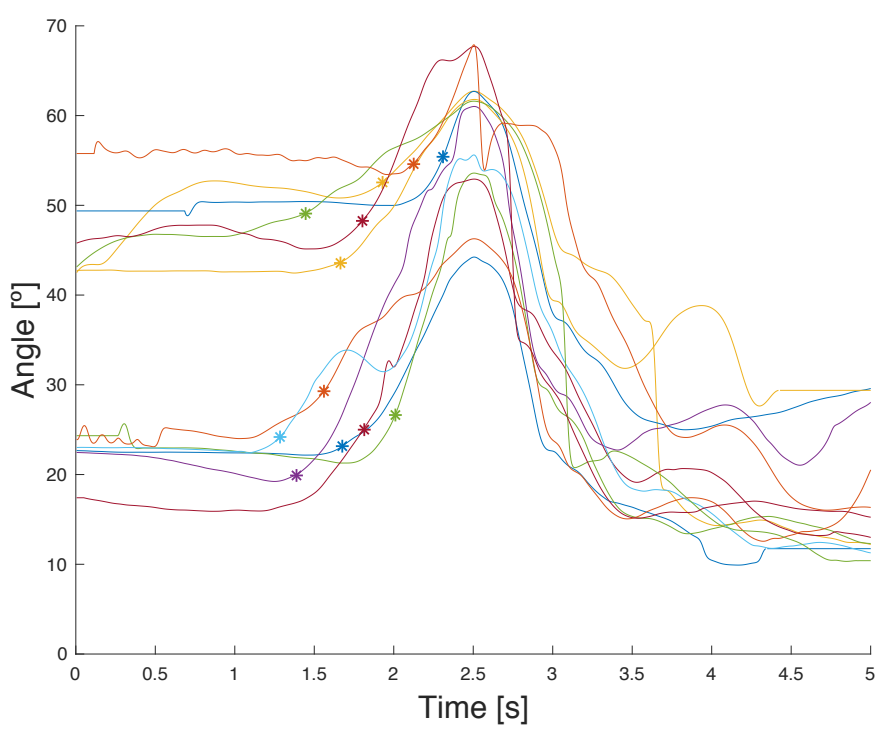

(a) Trunk angles from the motion capture system.

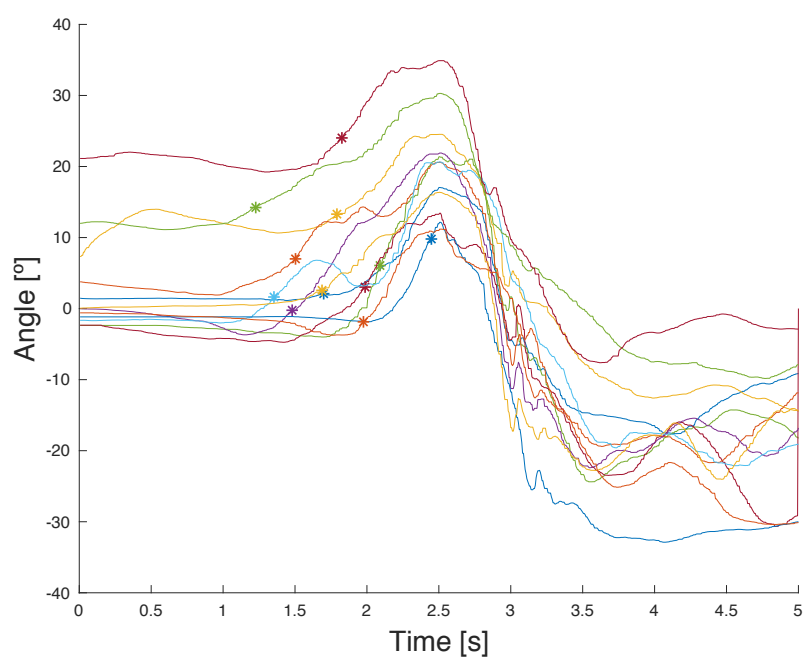

(b) Trunk angles from the IMU.

Figure 2: Trunk angles for all trials of subject B. The * marker indicates the moment the simulation was activated by the subject in each trial.

which may also be affected by the neck, but ignores the lower trunk movement, close to the hips.

We can see on Fig. 2a that the absolute angle varies substantially between trials of the same subject, which also makes it unsuitable to be used as trigger for the stimulation. However, the relative angle between the starting position and the moment of activation is more consistent, as can be seen on Fig. 3. Note that, despite the differences aforementioned between the two systems used, the relative angle seems similar between the two.

Having a high trunk angle correlation between trials on each subject is a strong indication that a device to automate the stimulation trigger is feasible. Table I shows low standard variations for the angles in which the stimulation was activated. That suggests that these angles collected by the IMU 


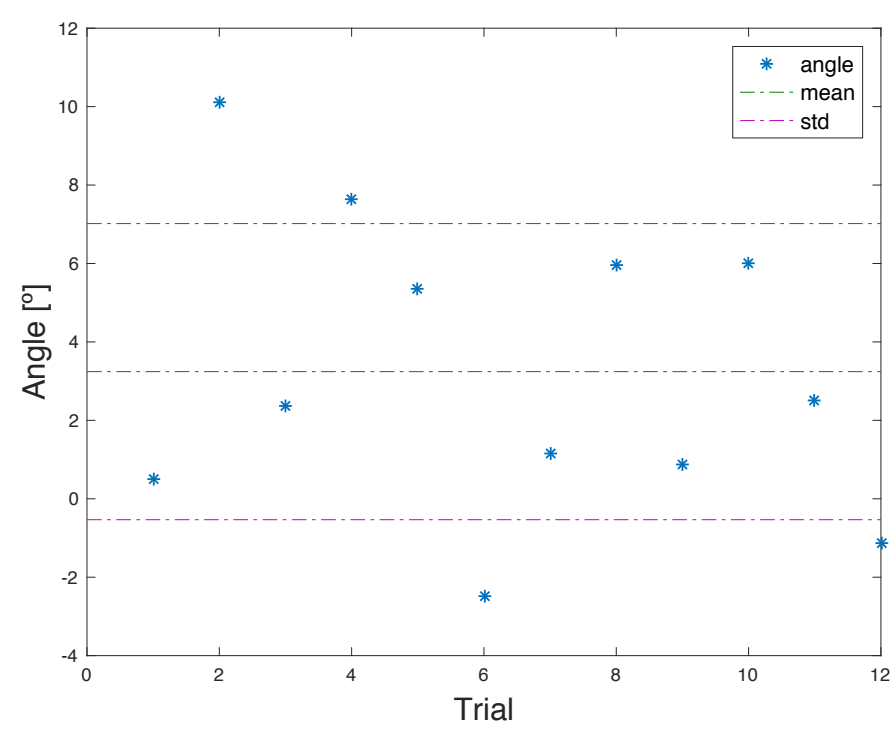

(a) Relative trunk angle from the motion capture system.

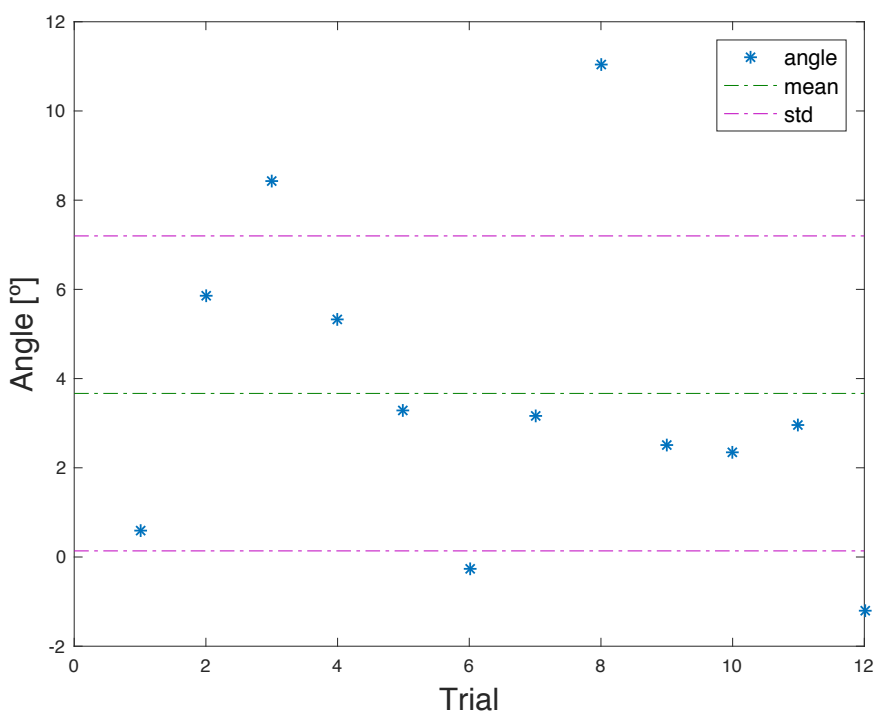

(b) Relative trunk angle from the IMU.

Figure 3: Trunk angle on the moment the stimulation was activated by the subject B related to the initial angle position. Each point depicts to one trial. The green dashed line is the average of all measurements, and the pink dashed lines represent one standard deviation up and down.

can be used to set a trigger, which must be calibrated for each individual, to activate the stimulation without the need of hand sensors or any other method.

It is important to point out that the transfers performed on this work were done without prior training, and the system performed reliably with all users. A real life application, however, would profit from some practicing, which we believe would further narrow the angle deviation. In addition, the relative angle would probably pose an easier learning curve for the user than something like the acceleration (second derivative), which would be harder to understand.

\section{CONCLUSION}

The IMU is a small device that could easily be adapted as a wearable device. The results found on this work indicate its data can be used to trigger the stimulation of lower limbs and assist SPT on paraplegic users. That would free their upper limbs to be used to support their remaining weight and balance, and possibly reduce the load, particularly on the shoulders. That, along with the high trunk angle correlation between trials, indicate that a reliable wearable device for such purpose if feasible.

\section{REFERENCES}

[1] G Desroches, D Gagnon, and S Nadeau. Magnitude of forward trunk flexion influences upper limb muscular efforts and dynamic postural stability requirements during sitting pivot transfers in individuals with. Journal of Electromyography and Kinesiology, 2013.

[2] D Gagnon, S Nadeau, L Noreau, JJ Eng, and D Gravel. Trunk and upper extremity kinematics during sitting pivot transfers performed by individuals with spinal cord injury. Clinical Biomechanics, 2008.

[3] Dany Gagnon, Alicia Koontz, Sara Mulroy, Debbie Nawoczenski, Emelie Butler-Forslund, Anna Granstrom, Sylvie Nadeau, and Michael Boninger. Biomechanics of Sitting Pivot Transfers Among Individuals with a Spinal Cord Injury: A Review of the Current Knowledge. Topics in Spinal Cord Injury Rehabilitation, 15(2):33-58, oct 2009.

[4] Dany Gagnon, Sylvie Nadeau, Luc Noreau, Janice J. Eng, and Denis Gravel. Electromyographic patterns of upper extremity muscles during sitting pivot transfers performed by individuals with spinal cord injury. Journal of Electromyography and Kinesiology, 19(3):509-520, jun 2009.

[5] Jovana Jovic, Christine Azevedo Coste, Philippe Fraisse, Sonia Henkous, and Charles Fattal. Coordinating Upper and Lower Body During FESAssisted Transfers in Persons With Spinal Cord Injury in Order to Reduce Arm Support. Neuromodulation: Technology at the Neural Interface, 18(8):736-743, dec 2015.

[6] Jovana Jovic, Sébastien Lengagne, Philippe Fraisse, and Christine Azevedo-Coste. Impact of Functional Electrical Stimulation of Lower Limbs during Sitting Pivot Transfer Motion for Paraplegic People. International Journal of Advanced Robotic Systems, 10(1):25, jan 2013.

[7] Padmaja Kankipati, Michael L. Boninger, Dany Gagnon, Rory A. Cooper, and Alicia M. Koontz. Upper limb joint kinetics of three sitting pivot wheelchair transfer techniques in individuals with spinal cord injury. The Journal of Spinal Cord Medicine, 38(4):485-497, jul 2015.

[8] Ana Claudia G Lopes, Claudia Ochoa-Diaz, Roberto S Baptista, Lucas O Fonseca, Charles Fattal, Christine Azevedo Coste, Antônio P.L. Bó, and Emerson Fachin-Martins. Electrical stimulation to reduce the overload in upper limbs during sitting pivot transfer in paraplegic: a preliminary study. European Journal of Translational Myology, 26(4):6223, aug 2016.

[9] EB Marsolais, A Scheiner, PC Miller, R Kobetic, and JJ Daly. Augmentation of transfers for a quadriplegic patient using an implanted FNS system. Case report. Spinal Cord, 1994.

[10] Julie O. Murphy, Musa L. Audu, Lisa M. Lombardo, Kevin M. Foglyano, and Ronald J. Triolo. Feasibility of closed-loop controller for righting seated posture after spinal cord injury. Journal of Rehabilitation Research and Development, 51(5):747-760, 2014.

[11] Paralyzed Veterans of America Consortium for Spinal Cord Medicine. Preservation of upper limb function following spinal cord injury: a clinical practice guideline for health-care professionals. The journal of spinal cord medicine, 28(5):434-70, 2005.

[12] RJ Triolo, C Bieri, J Uhlir, R Kobetic, and A Scheiner. Implanted FNS systems for assisted standing and transfers for individuals with cervical spinal cord injuries: clinical case reports. Arch Phys Med Rehab, 1996. 